\title{
Incidência, Mortalidade e Morbidade Hospitalar por Câncer em Crianças, Adolescentes e Adultos Jovens no Brasil: Informações dos Registros de Câncer e do Sistema de Mortalidade
}

doi: https://doi.org/10.32635/2176-9745.RBC.2018v64n3.56

Incidence, Mortality Rates and Hospital Morbidity for Children, Adolescents and Young Adults in Brazil: the Information from Cancer Registry is in the Mortality System

Incidencia, Mortalidad y Morbilidad Hospitalaria por Cáncer en Niños, Adolescentes y Adultos Jóvenes en el Brasil: Informaciones de los Registros del Cáncer y del Sistema de Mortalidad

\author{
Instituto Nacional de Câncer José Alencar Gomes da Silva. Rio de Janeiro: INCA; 2016. 412p. \\ ISBN 978-85-7318-318-4 (versão impressa) \\ ISBN 978-85-7318-315-3 (versão eletrônica)
}

Marceli de Oliveira Santos ${ }^{1}$

A primeira publicação sobre câncer infantojuvenil no Brasil ocorreu no ano de 2008 e reunia informaçóes sobre o perfil da morbimortalidade por câncer em crianças e adolescentes. Esta publicação representou um marco para o câncer em crianças e adolescentes, trazendo o tema à discussão, ampliando o conhecimento da realidade brasileira e a importância da utilizaçáo e divulgação dessa informação ${ }^{1}$.

A segunda publicação do gênero, lançada em 2017, amplia o escopo para a população de adultos jovens e incorpora na análise as informaçōes sobre morbidade hospitalar, o que permite conhecer o perfil da assistência oncológica no Sistema Único de Saúde (SUS) para essa populaçáo.

O câncer em crianças, adolescentes e adultos jovens (entre 0 e 29 anos) tem impacto significativo na saúde pública brasileira: é a segunda causa de morte no Brasil, sendo a primeira causa de morte por doença. É considerado raro, quando comparado com o câncer em adultos. O número de casos novos (incidência) representa de $2 \%$ a $4 \%$ de todos os tumores malignos.

Nas crianças menores de um ano, a incidência difere clínica e biologicamente daquelas que acometem as com mais idade. No Brasil, o percentual mediano correspondeu a 6\% de todos os tumores diagnosticados até 14 anos; enquanto, no mundo, esse percentual é de $10 \%{ }^{2,3}$. A incidência mediana foi de 126,58/1 milhão, com predominância do sexo masculino, sendo as leucemias as mais incidentes, seguidas dos tumores do sistema nervoso central (SNC). Destaca-se ainda um número expressivo de neoplasias não identificadas, refletindo a dificuldade diagnóstica. Em crianças e adolescentes, o Brasil segue o perfil de outros países, com maior frequência de leucemias em ambos os grupos etários (33\%, de 0 a 14 anos, e 26\%, de 0 a 19 anos). No grupo de 0 a 14 anos, os tumores do SNC ocupam a segunda posição (16\%), semelhante aos países desenvolvidos. Já em crianças e adolescentes (0 a 19 anos), os linfomas ocupam a segunda posição (14\%), assemelhando-se ao perfil de países em desenvolvimento. A incidência mediana para esse grupo foi de 139,99 casos por milhão, com picos nas faixas etárias de 1-4 e 15-19 anos. Os tipos de câncer mais incidentes foram as leucemias (37,75/1 milhão); linfomas (18,31/1 milhão) e tumores de SNC (18,08/1 milhão $)^{4-6}$.

A mortalidade por câncer infantojuvenil correspondeu à principal causa de morte por doença. Entre 0 a 14 anos, a taxa média de mortalidade foi de 32,07/1 milhão; enquanto, para 0 a 19 anos, o risco de morte representou 44,25/1 milhão. A faixa etária de 15 a 19 anos apresentou o maior risco de morte (54,01/1 milhão), sendo o grupo de 5 a 9 anos o de menor risco. As leucemias (14,94/1 milhão) foram as mais frequentes, seguidas pelos tumores do SNC (10,26/1 milhão) e tumores ósseos (3,14/1 milhão). A estimativa de sobrevida para o país alcança $64 \%$, bem diferente dos países desenvolvidos, onde atinge mais de $80 \%{ }^{6}$. Distinta também é a variação regional (de 50\% na Região Norte a 75\% na Região Sul).

${ }^{1}$ Instituto Nacional de Câncer José Alencar Gomes da Silva (INCA). Rio de Janeiro (RJ), Brasil. Orcid iD: https://orcid.org/0000-0001-5197-2019 Endereço para correspondência: Rua Marques de Pombal, 125 - Centro. Rio de Janeiro (RJ), Brasil. CEP 20230-240. E-mail: msantos@inca.gov.br. 
O câncer infantojuvenil correspondeu a 3\% de todas as neoplasias atendidas pelos hospitais de referência para tratamento de câncer no SUS, sendo os mais frequentes as leucemias (30,6\%), linfomas (16,6\%) e SNC (9,3\%). Em sua maioria, foram assistidos em hospitais habilitados para oncologia pediátrica; entretanto, na faixa etária entre 15 e 19 anos, esse percentual correspondeu a menos de 60\%. A análise do tempo decorrido entre consulta, diagnóstico e início do tratamento é uma importante medida para identificar as dificuldades de acesso do paciente com câncer à assistência especializada, seja pela demora no início do tratamento ou na confirmação diagnóstica. O tempo decorrido entre a primeira consulta e o diagnóstico foi de até 30 dias em $84 \%$ dos casos e de $75 \%$ entre o diagnóstico e o início do tratamento para o mesmo período de tempo. Com relação aos pacientes que chegaram sem diagnóstico e sem tratamento, $81 \%$ deram início ao seu tratamento em até 30 dias; dos que chegaram com diagnóstico e sem tratamento, $67 \%$ iniciaram em 60 dias ou mais, mostrando provavelmente a dificuldade de acesso daqueles pacientes que receberam o diagnóstico em outras unidades náo especializadas.

A publicaçáo também apresenta, de forma inédita, a situação dos adultos jovens. Esse grupo diferencia-se tanto das crianças quanto dos adultos e a incidência na faixa etária de 15 a 30 anos é 2,7 vezes maior do que em crianças menores de 15 anos $^{7}$. Diferencia-se também quanto aos tipos de câncer, sendo os linfomas e os carcinomas mais frequentes nesse grupo. Há um predomínio do sexo feminino em razão da alta incidência de tumores do colo do útero e tireoide. As principais causas de morte foram as leucemias e tumores do SNC. A faixa etária de maior risco foi a de 25 a 29 anos (87,85/1 milhão), destacando-se o câncer do colo do útero como principal causa de morte nessa faixa etária. Nesse grupo, não se observaram melhorias na sobrevida semelhantes à das crianças e adolescentes, em que a dificuldade de acesso e o atraso no diagnóstico são uma possível explicação.

$\mathrm{Na}$ assistência oncológica, os tumores mais frequentes foram os carcinomas e os linfomas. O tempo decorrido entre a primeira consulta e o diagnóstico foi de até 30 dias em $72 \%$ dos casos; entretanto, entre o diagnóstico e o início do tratamento, foi de mais de 60 dias em 75\% dos casos. Os pacientes que chegaram sem diagnóstico e sem tratamento, em $66 \%$ dos casos, iniciaram seu tratamento em até 60 dias, enquanto apenas $23 \%$ dos que chegaram com diagnóstico e sem tratamento foram tratados no mesmo tempo. O tempo mediano entre a consulta e o diagnóstico foi o dobro do observado para crianças e adolescentes, e quatro vezes maior entre o diagnóstico e o início do tratamento.

Conhecer o perfil do câncer nesse grupo específico no Brasil é relevante para motivar ações efetivas para o controle da doença e auxiliar no planejamento de serviços, já que evidencia a realidade. Informaçôes sobre os intervalos de tempo são fundamentais para subsidiar estratégias e otimizar os processos de diagnóstico e tratamento, questôes que impactam diretamente nas chances de cura do paciente. Aponta também desafios para superar a dificuldade diagnóstica, assinalando a importância da centralização, da revisão diagnóstica e do trabalho multidisciplinar em centros especializados.

\section{REFERÊNCIAS}

1. Instituto Nacional de Câncer José Alencar Gomes da Silva. Câncer da criança e adolescente no Brasil: dados dos registros de base populacional e de mortalidade. Rio de Janeiro: INCA; 2008.

2. Little, J. Introduction. In: Little, J. Epidemiology of childhood cancer. Lyon: IARC; 1999. p. 1-9. (IARC Scientific Publications; no. 149).

3. Birch JM, Blair, V. The epidemiology of infant cancers. Br J Cancer. 1992 Aug; 18 Suppl:S2-S4.

4. Howlader N, Noone AM, Krapcho M, Garshell J, Neyman N, Altekruse SF, et al., editors. SEER Cancer Statistics Review, 1975-2010. [Internet]. Bethesda, MD: National Cancer Institute; 2013. [update 2013 Jun 14; cited 2016 Jun 28]. Available from: https://seer.cancer.gov/csr/1975_2010/.

5. Parkin DM, Stiller CA, Draper GJ, Bieber CA. The international incidence of childhood cancer. Int J Cancer. 1988;42(4):511-520.

6. Ries LA, Smith MA, Gurney JG, Linet M, Tamra T, Young JL, et al, editors. Cancer incidence and survival among children and adolescents: United States SEER program 1975-1995. Bethesda, MD: National Cancer Institute, SEER Program, 1999. (NIH Pub; no. 99-4649).

7. Bleyer A, O'Leary M, Barr R, Ries LAG, editors. Cancer epidemiology in older adolescentes and young adults 15 to 29 years of age: including SEER incidence and survival: 1975-2000. Bethesda, MD: National Cancer Institute, 2006. (NIH Pub; no. 06-5767) 